\title{
HIPERGLICEMIA PÓS-TRANSPLANTE RENAL EM CRIANÇAS COM CISTINOSE'
}

\author{
After Kidney Transplantation Hyperglycemia in Child with Nephropathic Cystinosis ${ }^{1}$ \\ Liliane Cury Prates', Sumara Zuanazi Pinto Rigatto², Cíntia A Santana ${ }^{3}$, Simone M Nascimento ${ }^{3}$, Daniela G Carvalho ${ }^{3}$, \\ Anna Cristina G. Brito', Lívia C Oliveira ${ }^{3}$, Vera Maria Santoro Belangero ${ }^{4}$
}

\section{RESUMO}

Objetivo: Relatar a ocorrência de hiperglicemia em crianças com cistinose após transplante renal. Métodos: Revisão dos casos de transplantes renais pediátricos realizados no serviço. Resultado: A ocorrência de hiperglicemia após transplante renal é maior quando a causa da insuficiência renal é cistinose quando comparado com outras etiologias. Conclusão: Necessidade de protocolo imunossupressor especial em cistinóticos com menor efeito hiperglicemiante.

Descritores: Transplante Renal, Hiperglicemia, Cistinose, Criança.

Grau Acadêmico:

1. Mestre em Pediatria

2. Professor Doutor em Pediatria

3. Residente de Nefrologia Pediátrica

4. Professor Departamento de Pediatria

Instituição:

Serviço de Nefrologia Pediátrica - FCM UNICAMP - Campinas - SP - Brasil

Correspondência:

Liliane Cury Prates

Av. Presidente Kennedy, 333 apto. 81

CEP 13405-010 - Piracicaba - SP

Fax: $55113432-3244$

E-mail: lilianecprates@terra.com.br

Recebido em: 29/12/2005

\section{INTRODUÇÃO}

Cistinose é uma doença hereditária com acúmulo de cistina nos lisossomos em vários órgãos, levando à Síndrome de Fanconi e posterior insuficiência renal crônica (IRC), hipotireoidismo, dificuldades visuais, manifestações de sistema nervoso central, envolvimento de baço, fígado e pâncreas. $\mathrm{O}$ uso de cisteamina posterga a IRC, mas a droga ainda tem disponibilidade reduzida em nosso meio. $\mathrm{O}$ acometimento do pâncreas tende a ocorrer após a segunda década, mas hiperglicemia pode surgir mais precocemente após fatores facilitadores. ${ }^{1}$

\section{OBJETIVO}

Relatar a ocorrência de hiperglicemia em crianças com cistinose após transplante renal.

\section{MÉTODOS}

Dos 85 transplantes renais em 78 crianças, de cinco a 18 anos, do HCUNICAMP, no período de 1991 a 2005, seis apresentavam cistinose e realizaram sete transplantes, apresentados a seguir, na Tabela 1:

Tabela 1: Características gerais dos pacientes com cistinose, transplantados renais, segundo idade, sexo, imunossupressão.

\begin{tabular}{lll}
\hline Nome & Idade(anos) $-\mathbf{S e x o}(\mathbf{M} / \mathbf{F})$ & Imunossupressão \\
\hline AO & $10 / \mathrm{M}$ & TAC,MMF,P \\
TDPM & $6 / \mathrm{F}$ & TAC,AZA,P \\
AGO & $9 / \mathrm{M}$ & TAC,MMF,P \\
AAT & $6 / \mathrm{M}$ & CSA,AZA,P \\
AAT & $10 / \mathrm{M}$ & CSA,AZA,P \\
THCO & $12 / \mathrm{F}$ & TAC,MMF,P \\
AAO & $10 / \mathrm{M}$ & CSA,MMF,P \\
\hline
\end{tabular}

P: prednisona; TAC: tacrolimo; CSA: ciclosporina neoral; MMF: micofenolato mofetil; AZA: azatioprina 
A imunossupressão utilizada no serviço consta de prednisona-P (dose inicial $2 \mathrm{mg} / \mathrm{Kg} / \mathrm{dia}$, com redução gradual à dose de $5 \mathrm{mg}$ com seis meses), agente antiproliferativo (MMF dose inicial de $30 \mathrm{mg} / \mathrm{kg} / \mathrm{dia}$ ou, se efeitos colaterais deste, AZA $2 \mathrm{mg} / \mathrm{kg} / \mathrm{dia}$ ), e inibidor de calcineurina (CSA $7 \mathrm{mg} / \mathrm{kg} / \mathrm{dia}$ nos transplantes realizados antes de julho de 2001 e TAC 0,2 a $0,4 \mathrm{mg} / \mathrm{kg} /$ dia após esta data).

\section{ANÁLISE ESTATÍSTICA}

Para avaliação da significância da incidência de hiperglicemia em pacientes com e sem cistinose foi aplicado o teste do $\mathrm{X}^{2}, \operatorname{com} \mathrm{p}<0,05$.

\section{RESULTADOS}

De sete transplantes realizados em pacientes com cistinose, três apresentaram hiperglicemia de um mês a oito anos após o transplante (pacientes AO, TDPM, AGO que usavam tacrolimo) e necessitaram insulina para controle, enquanto dos 78 de outras etiologias, apenas uma criança apresentou hiperglicemia (etiologia da IRC: displasia renal, usando ciclosporina), que foi controlada com dieta $(\mathrm{p}=0.001)$.

\section{DISCUSSÃO}

O transplante renal elimina a deposição de cistina no rim transplantado, prolonga a sobrevida do paciente com cistinose, mas o acúmulo de cistina continua em outros órgãos, incluindo o pâncreas, sendo o Diabetes Mellitus (DM) uma complicação tardia da cistinose, ocorrendo geralmente na segunda década de vida. ${ }^{2,3,4}$ No entanto, DM pode ser precipitado pelo uso de altas doses de corticóide e/ou pelo uso dos inibidores da calcineurina (INC), especialmente pelo tacrolimo, que está associado com maior incidência de DM em transplantes pela inibição da transcrição do gene da insulina, com diminuição da produção e secreção de insulina. ${ }^{5}$ Sendo assim, pacientes com cistinose transplantados somam fatores de risco para DM.

\section{CONCLUSÃO}

Devido ao risco de DM em pacientes com cistinose, sugere-se protocolo especial de imunossupressão pós-transplante com menor efeito hiperglicemiante como suspensão precoce de corticóide e/ou não utilização de tacrolimo.

\section{ABSTRACT}

Objective: The present article reports the occurrence of hyperglycemia in cystinotic children who underwent renal transplant. Methods: Review on the pediatric renal transplantation cases occurred at this institution. Results: The rate of occurrence of pos-transplant hyperglycemia is higher whenever the cause of the renal failure is Cystinosis compared to other causes. Conclusion: It suggests that the immunosuppressive protocol in cystinotic patients should be different with lower hyperglycemic effect.

Keywords: Kidney Transplantation, Hyperglycemia, Cystinosis, Child.

\section{REFERÊNCIAS}

1. Filler G, Amendt P, von Bredow MA, Rohde W, Ehrich JH. Slowly deteriorating insulin secretion and C-peptide production characterizes diabetes mellitus in infantile cystinosis. Eur J Pediatr. 1998 Sep;157(9):738-42.

2. Filler G, Franke D, Amendt P, Ehrich JH. Reversible diabetes mellitus during growth hormone therapy in chronic renal failure. Pediatr Nephrol. 1998 Jun;12(5):405-7.
3. Robert JJ, Tête MJ, Guest G, Gagnadoux G, Niaudet P, Broyer M. Impaired glucose tolerance and insulin-dependent diabetes mellitus in infantile nephropathic cystinosis. In: Michel Broyer, editor. Cystinosis. Paris: Elsevier 1990. p. 56-62.

4. Fivush B, Green OC, Porter CC, Balfe JW, O’Regan S, Gahl WA. Pancreatic endocrine insufficiency in post transplant cystinosis. Am J Dis Child. 1987 Oct;141(10):1087-9.

5. Mayer AD et al. Multicenter randomized trial comparing tacrolimus (FK506) and cyclosporine in the prevention of renal allograft rejection: a report of the European Tacrolimus Multicenter Renal Study Group. Transplantation 1997;64(3):436-43.

JBT J Bras Transpl. 2005;8:425-426. 